\title{
Wideband Communication for Implantable and Wearable Systems
}

\author{
Mehmet Rasit Yuce, Member, IEEE, Ho Chee Keong, and Moo Sung Chae
}

\begin{abstract}
This paper presents the feasibility of applying an ultra-wideband (UWB) wireless scheme to both high data rate implantable neural recording and low data rate wearable biomedical applications. An extensive analysis on the UWB signal generation for biomedical application is discussed. A CMOS UWB transmitter has been designed, fabricated, and used in a high data rate neural recording system. A method to readily assemble a flexible UWB transmitter for wearable physiological monitoring system is also presented. An eight-channel low data rate recording system for monitoring multiple continuous electrocardiogram and electroencephalogram signals has been designed, and its test results are presented.
\end{abstract}

Index Terms-CMOS pulse generator, implantable electronics, ultra-wideband (UWB), wireless body area network (WBAN).

\section{INTRODUCTION}

$\mathbf{T}$ HERE HAS been a growing trend of employing wireless technologies for measuring physiological signals for both implantable and wearable systems. Two such areas that are of significant interest for deploying wireless technologies are the implantable multichannel neural recording system and wearable wireless body area network (WBAN).

Advances in microelectrode arrays (MEAs) and multichannel neural recording systems [1]-[6] improved the quality of the signals recorded, but at the expense of higher numbers of channels. Those systems, due to a large number of recording channels, naturally produce a huge amount of data that should be transmitted out of body to be processed or analyzed further. For example, a 128-channel neural recording system with 8-bit resolution at the Nyquist sampling rate generates $20 \mathrm{Mb} / \mathrm{s}$ when recording extracellular action potentials whose signal energy spectrum is up to $10 \mathrm{kHz}$. As the number of channel increases, the data rate will reach a speed of $100 \mathrm{Mb} / \mathrm{s}$ and higher. This large amount of data is desired to be transmitted to a receiver that is located outside the body wirelessly. Using wires is not preferable in neural recording applications because those wires restrict the movement and behavior of animals or humans to be observed, but

Manuscript received March 13, 2009; revised June 23, 2009. First published September 18, 2009; current version published October 14, 2009. The work of M. R. Yuce and H. C. Keong was supported by the Australian Research Council (ARC) under Discovery Projects DP0772929.

M. R. Yuce and H. C. Keong are with the School of Electrical Engineering and Computer Science, University of Newcastle, Callaghan, N.S.W. 2308, Australia (e-mail: mehmet.yuce@newcastle.edu.au; chee.ho@newcastle.edu.au).

M. S. Chae is with the Department of Electrical and Computer Engineering, University of California at Santa Cruz, Santa Cruz, CA 95064-1077 USA (e-mail:gomdori07@gmail.com).

Color versions of one or more of the figures in this paper are available online at http://ieeexplore.ieee.org.

Digital Object Identifier 10.1109/TMTT.2009.2029958 also increase the possibility of infections. Furthermore, wireless capability is also preferred at the cosmetic point of view.

The allowable channel bandwidth of the medical implant communication service (MICS): a wireless scheme approved for use in an implantable environment is only $300 \mathrm{kHz}$; it is unable to support the high data rate required. Current state-of-art wireless neural recording chip [4] is able to provide recording for 100 channels, but due to bandwidth limitation, it can only support one channel of raw data transmission. Therefore, it is quite obvious that there is an immediate need for higher bandwidth data transmission for neural recording telemetry systems.

As the FCC assigned the spectrum from 3.1 to $10.6 \mathrm{GHz}$ for unlicensed use of UWB devices to support high data rates, ${ }^{1}$ it provided an opportunity for a wideband wireless telemetry. Impulse radio UWB (IR-UWB) uses simple short pulses for sending data, it makes the transmitter design very simple, small area, and low power. This paper presents an IR-UWB transmitter that is designed in National's $0.35-\mu \mathrm{m}$ CMOS process and fabricated for neural recording application [7], [8].

Apart from implantable, deployment of wireless technology for wearable medical monitoring has improved patient quality of life and efficiency of medical staff. Aging population, shortage of medical staff, and high demand of hospital resources are problems faced in many countries around the world. A wireless medical sensor network facilitates remote monitoring and allows the medical staff to detect early symptoms. Timely medical intervention significantly improves the patient's chances of recovery. Several proprietary solutions based on Bluetooth, ZigBee, and wireless local area networks (WLANs) are available, but they are not optimized for body sensor networks and lacks interoperability. Therefore, there is a need for standardization to ensure interoperability and provides an optimized solution for WBANs. A task group (IEEE802.15.6) was formed in November 2007 to develop a standard for WBANs. The targeted data rate for WBANs is $10 \mathrm{Mb} / \mathrm{s}$. Low data rate UWB is one of the potential candidates, to overcome the bandwidth limitations of current narrowband systems, and to improve the power consumption and size. Another advantageous is that UWB does not have any interference on other wireless medical devices when used in a medical environment due its low-power transmission. An eight-channel wearable physiological signals monitoring system has been designed and tested to show the feasibility of low data rate UWB for a WBAN application.

\footnotetext{
${ }^{1}$ Federal Communications Commission (FCC), Washington, DC. [Online] Available: http://www.fcc.gov
} 


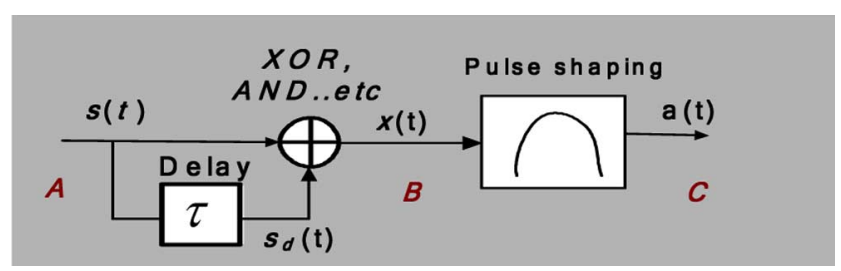

Fig. 1. Narrow pulse generator.

\section{UWB SignAL GENERATION FOR LOW-POWER BIOMEDICAL CIRCUITS}

There are two common transmitter categories used for UWB technology. The transmitters in the first category include a pulse generator and an up-converter that uses a mixer and a local oscillator (LO) to convert the baseband signal into the UWB band [9]. The transmitters in the second category consist of a short pulse generator and a pulse-shaping circuitry that makes the bandwidth of the generated pulses directly fall in the UWB band. In these transmitters, there is no need for a mixer and an LO, significantly reducing the complexity and power consumption of the transmitter [10]. Since the transmitter in our application does not require a complex multiple-access communication and the power consumption is the most critical design specification, the second type of transmitter design technique is preferred for biomedical system.

\section{A. UWB Pulse Generation Techniques}

There are various methods to generate UWB pulses. Among all methods, using the delay-and-AND gate or delay-and-XOR gate is the least complex way in CMOS integrated circuit (IC) technology [11]. The delay unit can be realized using digital gates such as inverters, analog differential delay cells [12], flipflops, and controllable capacitors [13]. A general scheme for such pulse generations is given in Fig. 1. Herein we apply a bandpass filter as a pulse shaper due to its simplicity, which is desired for low-power biomedical applications.

\section{B. Analysis of Band-Limited UWB Pulses}

In this section, we will first analyze pulse generation schemes in both the time and frequency domains. The method described here can be applied to different applications in order to meet the spectral mask of the UWB band.

The data signal $s(t)$ and the delayed replica $s_{d}(t)$ are passed through an XOR gate or an AND gate to obtain a UWB narrow pulse $x(t)$ (e.g., $x(t)=S(t) . S(t-\tau)$ ). A narrowband square wave can be represented by

$$
x(t)=\sum_{n=-\infty}^{\infty} g_{T}\left(t-n T_{b}\right)
$$

where $T_{b}$ is the bit period and

$$
g\left(t-n T_{b}\right)=\left\{\begin{array}{cc}
A & n T_{b}<t \leq\left(n T_{b}+\tau\right) \\
0 & \left(n T_{b}+\tau\right)<t \leq(n+1) T_{b}
\end{array}\right\}
$$

where $A$ is the amplitude of the pulse and $\tau$ is the width of the

$$
x(t)=\frac{A \tau}{T_{b}}+\frac{2 A \tau}{T_{b}} \sum_{k=1}^{+\infty} \frac{\sin \left(\pi \frac{k \tau}{T_{b}}\right)}{\left(\pi \frac{k \tau}{T_{b}}\right)} \cos (k w t)
$$

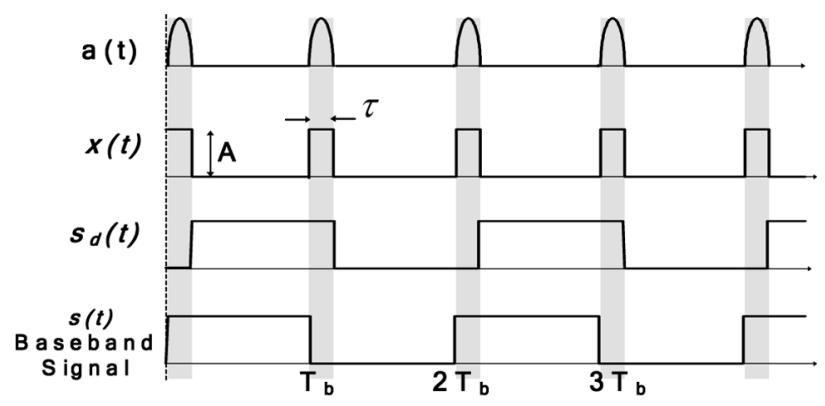

Fig. 2. Timing diagram for UWB pulse generation.

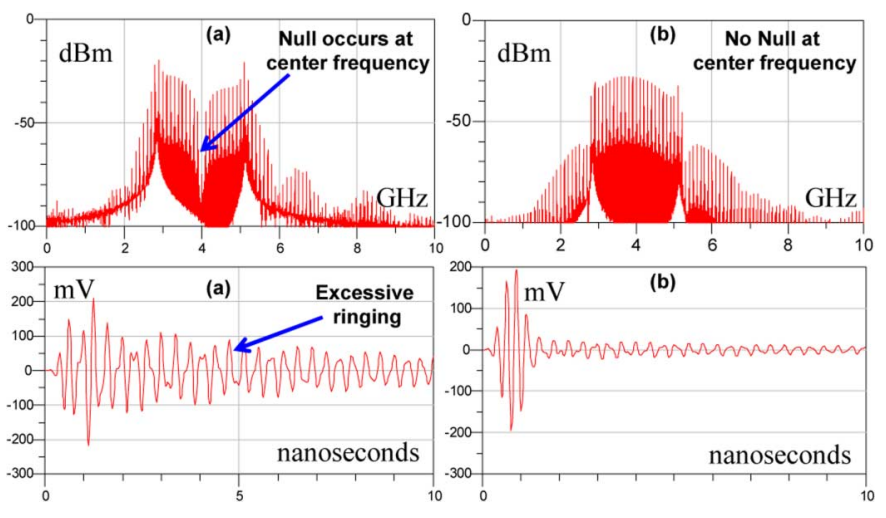

Fig. 3. Effects of null on UWB. (a) Signal with null. (b) Signal without null.

UWB pulses obtained from the delay element, as depicted in Fig. 2. Note that when code schemes like Manchester nonreturn zero (NRZ) is used for data, there will always be a transition repeated every bit period $T_{b}$. Assuming there is a square pulse repeated in every bit period, the Fourier series of the signal in (1) is given as (3) [14]. The signal includes a dc term and the fundamental frequency together with harmonic frequencies. The least complex method of generating a UWB compliant pulse is passing the narrow square pulse through a pulse-shaping circuitry, as shown in Fig. 2. The pulse-shaping circuitry can be a high-pass or bandpass filter. Since the square pulse is band-limited after passing through a bandpass filter, it can be represented by (4) as follows:

$$
x(t)=\frac{2 A \tau}{T_{b}} \sum_{k=n 1}^{n 2} \frac{\sin \left(\pi \frac{k \tau}{T_{b}}\right)}{\left(\pi \frac{k \tau}{T_{b}}\right)} \cos (k w t)
$$

where $\omega=2 \pi / T_{b}, n 1=\omega / \omega_{1}, n 2=\omega / \omega_{2}$, and $\omega_{1}$ and $\omega_{2}$ are the lower and upper cutoff frequencies of the bandpass signal.

The band-limited signal described by (4) assumes ideal zero rise and fall times, which is applicable for low-frequency system analysis, but in a high-frequency $(>1 \mathrm{GHz})$ scenario, finite rise/ fall time causes an additional null in the band of interest, as shown in Fig. 3. A null occurring in the band of interest may cause the signal to be unstable after going through a bandpass filter. In the worst case scenario, as illustrated in Fig. 3(a), when a deep null occurs at the center frequency [see Fig. 3(a)] of the transmission band, it causes the noise floor to increase significantly due to excessive ringing. 
A realistic pulse with finite rise and fall times can be represented by (5) as follows:

$$
\begin{aligned}
x(t)= & \frac{2 A\left(\tau+t_{r}\right)}{T_{b}} \sum_{k=n 1}^{n 2} \frac{\sin \left(\pi k \frac{t_{r}}{T_{b}}\right)}{\pi \frac{k t_{r}}{T_{b}}} \\
& \times \frac{\sin \left(\frac{\pi k\left(\tau+t_{r}\right)}{T_{b}}\right)}{\frac{\pi k\left(\tau+t_{r}\right)}{T_{b}}} \cos (k \omega t) \\
f_{c} t_{r}< & 0.5 \quad \text { where } \alpha=1,3,5,7,9, \ldots
\end{aligned}
$$

A finite rise time adds an additional null to the circuit. A null due to rise/fall times occurs every $1 / t_{r}$, the null arises from the pulsewidth and appears every $\left(\tau+t_{r}\right)$ interval. Therefore, the format of the transmitted pulse should be arranged to ensure that the null does not appear at the frequency band of interest. It is desirable to keep the spectrum peak of the sinc envelope at the center frequency. Satisfying these two criteria would enhance the signal-to-noise ratio (SNR) of the system.

The rise time can be optimized using (6), where 0.5 corresponds to a 4-dB loss. The peak of the spectrum (i.e., the sinc envelope) can be put at the center frequency by using (7), where $\alpha$ represents the sidelobes, " 1 " is the main lobe and " 3 " is the first sidelobe. Optimizing the nulls and peak of the spectrum enhances the performance of the transmitter, which is achieved by adjusting the pulsewidth.

Apart from controlling the pulsewidth and rise time, the power spectrum is optimized to meet the UWB emission mask by controlling both $A$ and $T_{b}$. Using (5), the amplitude of the spectral lines become inversely proportional to their frequencies (i.e., amplitudes $=2 A / k \pi$ ). Lower data rates contain more spectral lines, but are lower in amplitude. For example, for a system with a 2-GHz bandwidth spanning from 3.1 to $5.1 \mathrm{GHz}$ and a data rate of $100 \mathrm{Mb} / \mathrm{s}$, the maximum possible number of spectral lines is 21 and the amplitude is multiplied by a factor of $2 A / k \pi$, where $k$ ranges from 30 to 51 , while a system with a data rate of $10 \mathrm{Mb} / \mathrm{s}$ contains 200 spectral lines and $k$ ranges from 300 to 500 . Therefore, the FCC emission limit of $-41 \mathrm{dBm} / \mathrm{MHz}$ can be met by either increasing the pulse repetitive frequency (PRF) or increasing $A$.

Maintaining the data rate, while increasing the PRF (i.e., sending multiple pulses per data bit), results in a processing gain, and therefore, eliminates the need for a power amplifier, but high PRF system is limited by the FCC average power requirement, and therefore, is not optimized for a communication range. To optimize the communication range, the transmitted power should be close to both the peak power limit and average power limit. The relationship between the FCC's peak limit and average limit for a $1-\mathrm{MHz}$ bandwidth is illustrated in (8). The FCC peak limit for $1-\mathrm{MHz}$ resolution bandwidth is $-17 \mathrm{dBm}$ and the average limit is $-41-\mathrm{dBm}$ effective isotropic radiated power (EIRP). The pulsewidth $\left(\tau_{1}\right)$ for this system is determined by the bandpass filter. For example, a pulsewidth of $1 \mathrm{~ns}$, the optimal PRF to achieve a maximum peak power limit of $20 \mu \mathrm{W}$ for this system is $3.75 \mathrm{MHz}$. For a low PRF

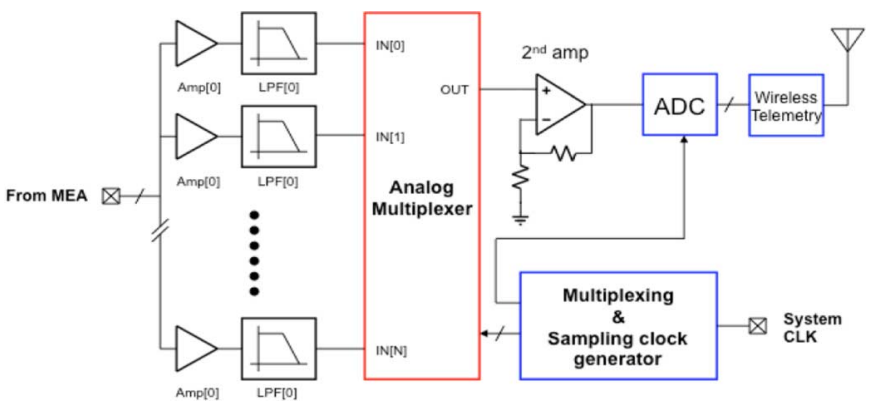

Fig. 4. Simplified block diagram of typical multichannel neural recording systems.

of $3.75 \mathrm{MHz}$, the processing gain is low. Therefore, a power amplifier is required to boost the transmission power (higher $A$ ) in order to be near to the emission limit. The use of a power amplifier would increase the power consumption. Therefore, there is a tradeoff between lower power consumption with the use of the processing gain and that of the communication range.

$$
P_{\text {peak }} \tau_{1}=P_{\text {ave }} T_{b}
$$

\section{UWB TRANSMITTER FOR NEURAL RECORDING}

A general multichannel wireless neural recording system consists of preamplifiers, filters, an analog multiplexer, a second amplifier, an analog-to-digital converter (ADC), and a wireless telemetry, as shown in Fig. 4. Preamplifiers are usually designed to have input dc blocking capacitors due to a large dc offset voltage present in electrode-electrolyte interface. The cutoff frequency of the low-pass filters following the preamplifiers are set to $10 \mathrm{kHz}$ for antialiasing because the signal spectrum of the extracellular action potential can be as high as $10 \mathrm{kHz}$. Time multiplexing is performed by the analog multiplexer and the second amplifier provides additional gain for the proper operation of the following ADC. The overall gain of the amplifiers is set to over $60 \mathrm{~dB}$ for the recording of extracellular action potentials whose typical amplitude is around $100 \mu \mathrm{V}$. Since all the recording channels share one second amplifier, the power consumption of the second amplifier is not critical even though it should have relatively high bandwidth and slew rate compared to the preamplifiers. The ADC converts the time multiplexed analog signal into digital data and then the digitized bit streams are fed to the wireless telemetry.

The resolution of the ADC is determined based on the SNR of the signal to be recorded, which is mostly affected by the noise from the recording electrodes [15]. For the extracellular recording with MEAs whose electrode-electrolyte interface impedance is $1 \mathrm{M} \Omega$, the SNR is around $40 \mathrm{~dB}$, resulting in a conservative 9-bit resolution of the ADC [8]. The sampling rate is set to $40 \mathrm{ksample} / \mathrm{s} / \mathrm{channel}$, which is four times higher than the cutoff frequency of the low-pass filters. Therefore, the raw data rate produced by the analog front-end with 128 recording channels is $46 \mathrm{Mbit} / \mathrm{s}$. However, this raw data should be expanded with redundant bits for channel separation and other purposes required by the wireless communications. Therefore, for 128 channel neural recording systems, the UWB telemetry 


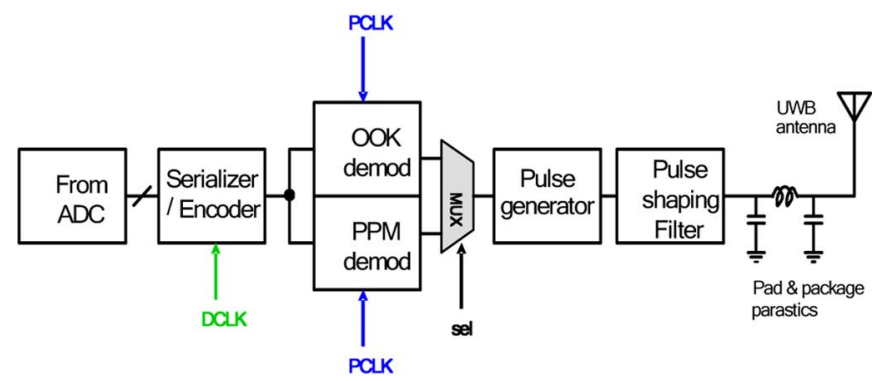

Fig. 5. Block diagram of IR-UWB transmitter for wireless telemetry.

should be able to support a data rate up to $100 \mathrm{Mb} / \mathrm{s}$ for the lossless simultaneous recording [7], [8].

Power consumption is also critical in implantable devices because the excessive heat produced by the device can easily give damages to the tissue surrounding the implanted devices. Even a temperature increase by $1{ }^{\circ} \mathrm{C}$ will cause serious heat damages for cortical signal recordings. The wireless telemetry unit is the most power-consuming circuit in wireless neural recording systems and for this reason, IR-UWB is the best choice because the IR-UWB transmitter consumes very small power and is easy to integrate on-chip by the nature [8].

\section{A. CMOS UWB Transmitter Design}

For pulse generation, the method described in Section II is followed. Fig. 5 is the block diagram of the IR-UWB transmitter. The first stage of the transmitter is an encoder. The encoder is used to convert the baseband data into different formats (e.g., NRZ Manchester). It can also be used to enable the receiver to recover clocks directly from the encoded data, as well as to distinguish the data from different channels. After configuring the transmitter to a modulation scheme, the encoded data is then passed to a narrow pulse generator. The pulse generator circuit used is shown in Fig. 6. In this circuit, a pulse width is adjusted from 200 to $900 \mathrm{ps}$ by the external control voltage $V c$. Generated pulses are passed through the pulse-shaping filter to fit them into the FCC emission mask and to eliminate the transmission of unnecessary frequencies. Unlike other UWB applications, power amplifiers are not necessary due to the low transmitted power and the short-distance range in neural recording applications. Instead, a wideband matching filter (acting as a bandpass filter) is used to regulate the transmitted power. The matching circuit together with the parasitic components from the output pad and the package pin contributes extra derivation (e.g., further filtering in frequency domain) to the UWB Gaussian pulse that makes the signal more suitable for an UWB transmission [10], [12].

Our transmitter can be configured to different pulse modulation schemes: on-off keying (OOK), pulse-position modulation (PPM), and binary phase-shift keying (BPSK). A signal OOK_in is generated by passing the NRZ and Manchester NRZ baseband signals through an AND gate. As shown in Fig. 7, when the signal OOK_in is given to the pulse generator circuit depicted in Fig. 6, $x(t)$ will be an OOK modulated signal [see Fig. 7(d)]. During bit "1," a pulse is transmitted, and meanwhile there is no pulse during bit " 0 ." The PPM UWB signal is generated by using the Manchester NRZ signal and the OOK UWB

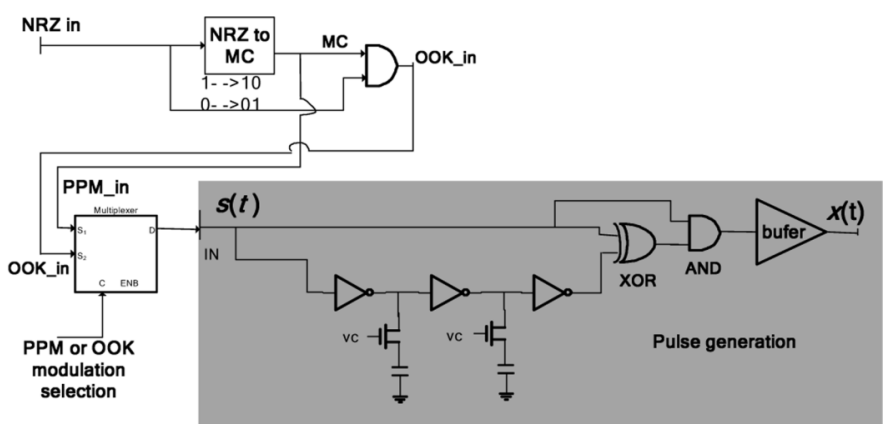

Fig. 6. Circuit used for pulse generation and modulation selection.

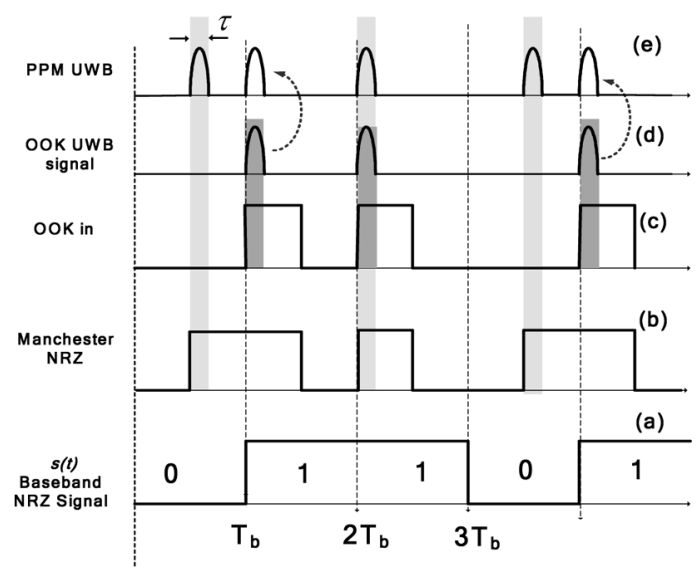

Fig. 7. Time diagram for PPM and OOK modulation.

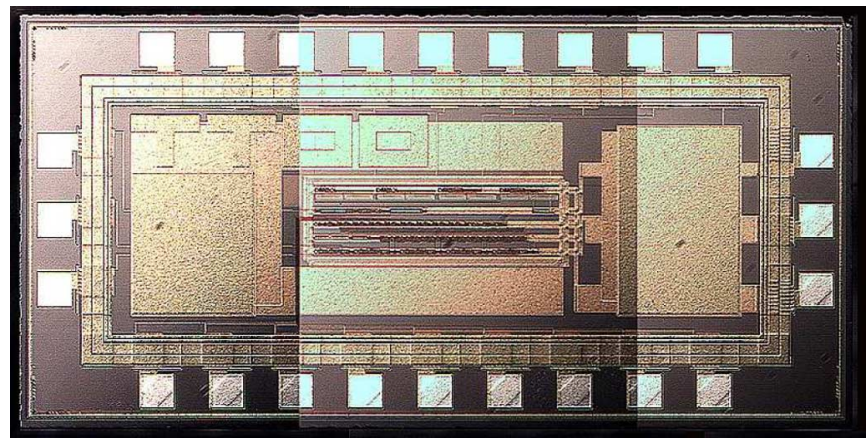

Fig. 8. Fabricated UWB transmitter chip.

signal. First the Manchester NRZ is passed through the pulse generation circuit. The resulted narrow pulses are added (using OR gate) to the OOK UWB [see Fig. 7(d)] to obtain PPM UWB in Fig. 7(e). As can be seen, the pulse position for bit "1" is different than that of bit " 0 ." The bits are positioned such that the bit detection will be easier at the receiver site. To generate a BPSK signal, the pulse is simply inverted by $180^{\circ}$ when the bit is " $0 . "$

\section{B. Circuit Measured Result}

The transmitter circuit in Fig. 5 is designed, laid out, and fabricated using National's $0.35-\mu \mathrm{m}$ CMOS process and the chip photograph is shown in Fig. 8. The circuit consumes $24 \mu \mathrm{A}$ for a $5.12-\mathrm{Mb} / \mathrm{s}$ OOK UWB signal generation and $243 \mu \mathrm{A}$ for a $100-\mathrm{Mb} / \mathrm{s}$ OOK UWB signal generation at the power supply 


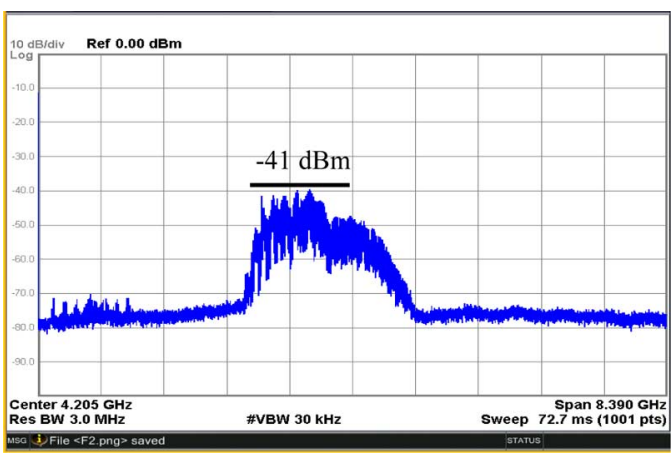

Fig. 9. Spectrum of a transmitted UWB signal.

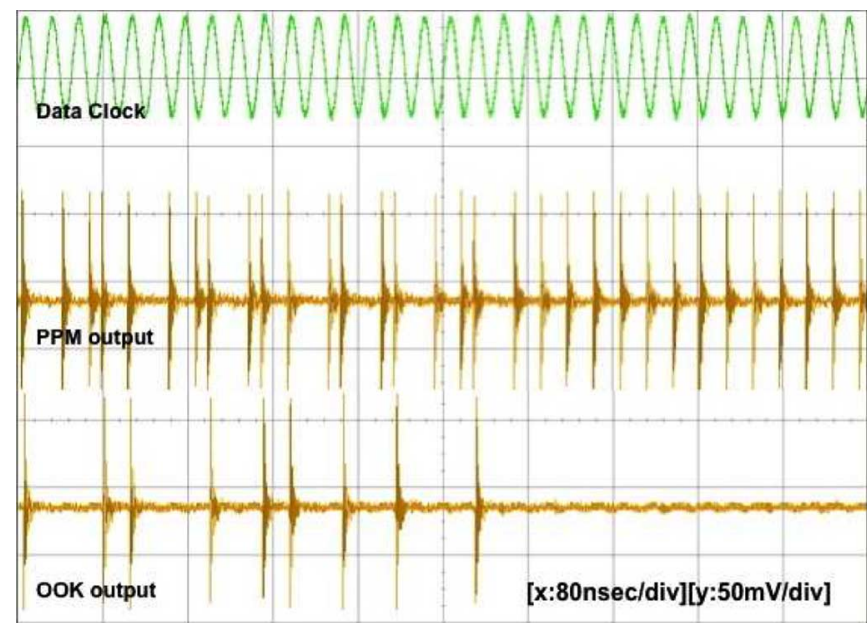

Fig. 10. Outputs time waveforms measured with oscilloscopes.

level of $3.3 \mathrm{~V}$. During the PPM modulation, the circuit consumes about twice as that of OOK because both " 0 " and " 1 " require pulse generation. Although PPM consumes more power than OOK in this IR-UWB implementation, it has an advantage of easier synchronization at the receiver side because every symbol always has a pulse.

Unlike other UWB applications, in our neural recording system, the implanted device does not require a receiver circuit. The transmission is unidirectional, as the information needs to be recorded and monitored only. This greatly simplifies the complexity of the implanted device and increases its lifetime. Pulse shaping is performed by sending the generated pulses through a 4-GHz bandpass filter (BPF) that has a bandwidth of $1 \mathrm{GHz}$. The measured UWB compliant spectrum at the output of the transmitter is shown in Fig. 9 and the time waveform at the output of the transmitter with an oscilloscope is presented in Fig. 10, respectively. The inter-symbol interference (ISI), which is heavily dependent upon the characteristics of the package, had a critical effect on the maximum data rate achievable at the transmitter side in our experimental setup. On the receiving end, the high-speed receiver is built from off-shelf components using high-performance RF ICs (i.e., low-noise amplifiers (LNAs), mixers) and high-speed field-programmable gate arrays (FPGAs). Details of the receiver prototype system developed to test the concept defined in this paper will be covered in Section IV. Fig. 11 shows the measured result of the

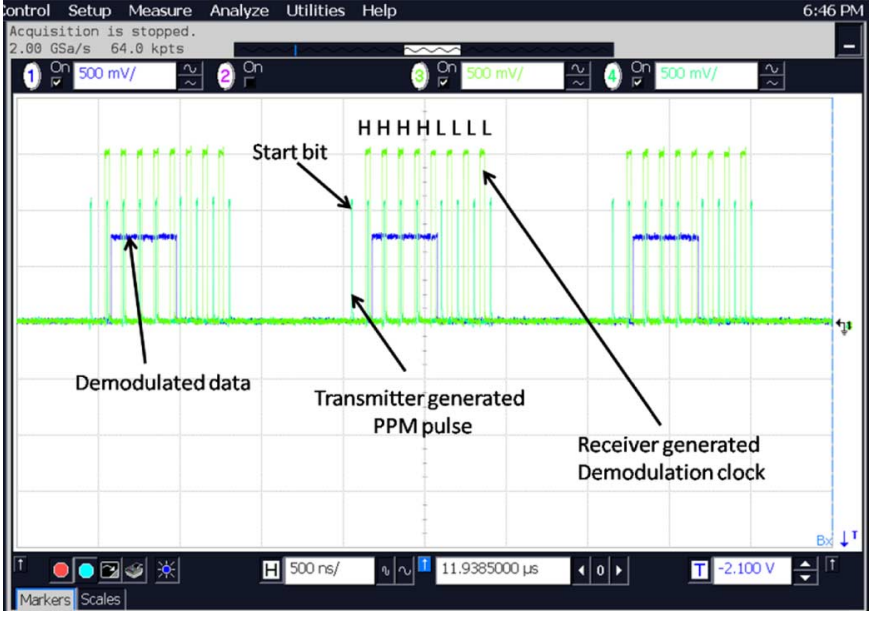

Fig. 11. Generated PPM pulses with demodulated result.

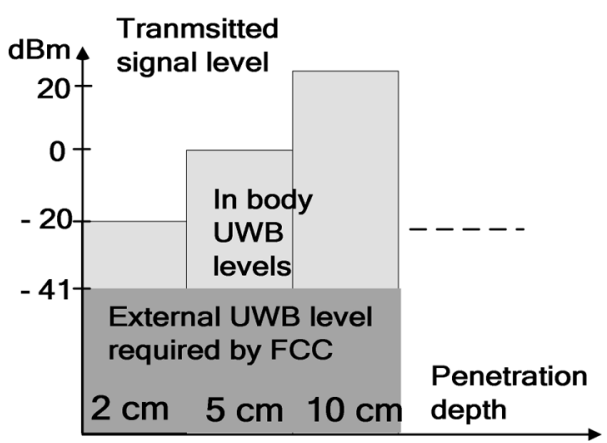

Fig. 12. Power level arrangement for transmitted UWB signal in body.

generated PPM pulses together with the receiver demodulation clock and the demodulated data. UWB chip antennas (Fractus fr05-107) with the physical dimension of $10 \times 10 \times 0.8 \mathrm{~mm}^{3}$ have been used for both Tx and Rx. The result is obtained based on the transmission distance of $1 \mathrm{~m}$ in a direct line-of-sight environment. Another test is carried out by enclosing the transmitter antenna in salt-reduced corned beef silverside to simulate the implantable environment. Fig. 12 illustrated the result of the test, the deeper the penetration depth (i.e., the deeper the transmitter is inserted into the human body), the higher the transmission power required. Therefore, implantable devices should be able to transmit more than $-41 \mathrm{dBm} / \mathrm{MHz}$ while meeting the FCC requirement.

\section{Receiver Implementation}

The received signal is passed through a bandpass filter centered at $4 \mathrm{GHz}$ with $1-\mathrm{GHz}$ bandwidth to remove the narrowband interfering signals. Three LNAs, each with a gain of $14 \mathrm{~dB}$, is used to amplify the signal, which gives a total gain of $42 \mathrm{dBm}$. The amplified signal goes through a diode detector, which translates the high-frequency components to their low-frequency components. The recovered low-frequency components are passed through a low-pass filter to form an envelope of the data signal. The signal is further amplified by an op-amp circuit by $60 \mathrm{~dB}$ before sending to the ADC.

The recovered data is converted to digital signal using 12-bit ADC embedded in the Stratix II development board. 


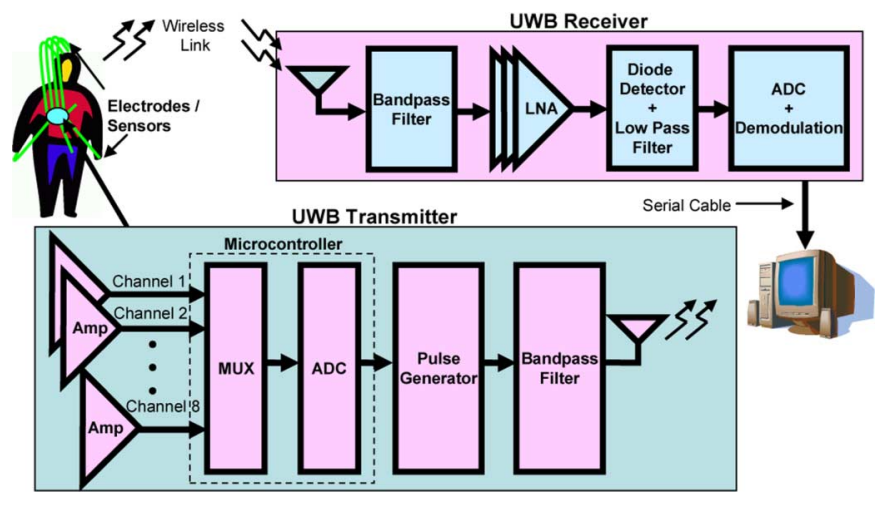

Fig. 13. Multichannel UWB monitoring system block diagram.

The demodulation is carried out with an FPGA chip running at $100 \mathrm{MHz}$ (capable of demodulating data of $50 \mathrm{Mb} / \mathrm{s}$ ). The transmitter sends data in block of eight pulses with an additional pulse as a start bit. There is a blank interval between each transmission, as shown in Fig. 10. Demodulation is performed by synchronizing the demodulation clock with the start bit. Once synchronized, the FPGA will generate eight clock cycles that is aligned with pulse position for bit " 1. " Pulses that occurs within the demodulation clock period is bit " 1 ," those outside are bit " $0 . "$ After the eighth cycle, the FPGA will goes into sleep mode and wake up just before the next start bit.

\section{Eight-Channel Wearable Medical MONITORING SYSTEM}

In this section, an eight-channel UWB recording system for monitoring of multiple continuous electrocardiogram (ECG) and electroencephalogram (EEG) signals, as shown in Fig. 13, is presented. One of the major differences between an implantable and wearable body sensor monitoring system is the location of the receiver. The receiver for an implantable device is typically placed at a fixed location near to the where the transmitter is implanted, but for a wearable monitoring system, the distance between the transmitter and receiver is constantly changing due to movement of the body. As the distance gets further or when the direct path is blocked, more gain is required for the signal to be transmitted reliably. Therefore, the transmitter need to adjust the gain constantly to ensure reliable transmission is achieved. Gain can be increased using either by sending more pulses per bit or increasing the pulse energy. A pulse generator that ultilizes the band-limited concept described in Section II-B, to adjust the gain by changing the PRF and amplitude is assembled using readily available commercial-off-the-shelf (COTS) components. A microcontroller PIC18F2320 is included in the transmitter to perform the task of multiplexing, analog to digital conversion, and adding the control bits. The use of COTS components helps to reduce the prototyping time and allows for greater flexibility in changing the transmitter configuration to adapt to various applications.

\section{A. UWB Pulse Generator for Wearable System}

Fig. 14 shows the block diagram of the proposed COTS pulse generator. The pulse generator is able to generate pulse width ranging from 300 ps to 4 ns by adjusting the VDDB1

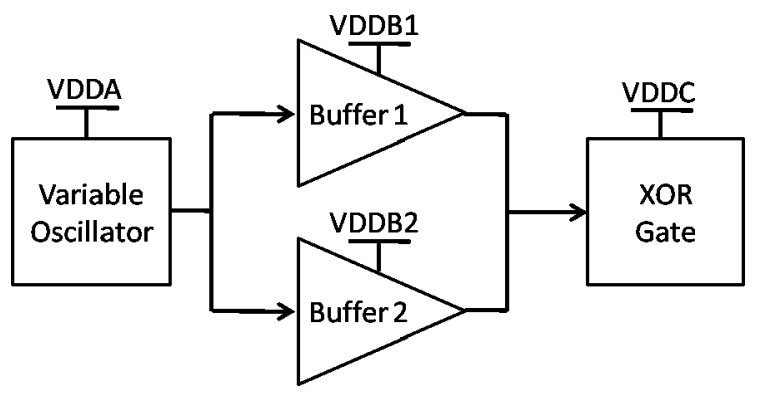

Fig. 14. Block diagram of proposed COTS UWB pulse generator.

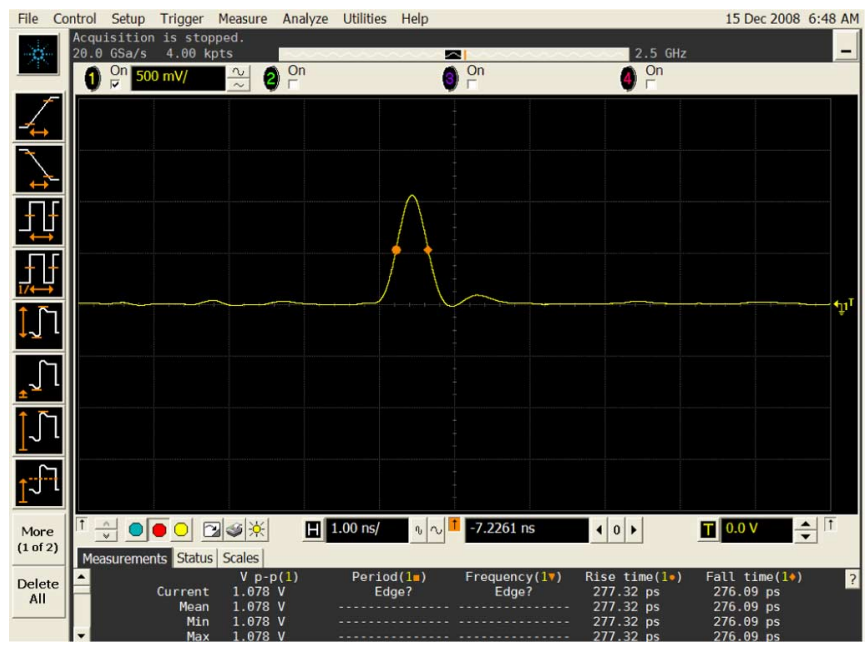

Fig. 15. UWB pulse generated by COT transmitter.

and VDDB2. The propagation delay of a buffer is given in (9) [16]. The load capacitance $\left(C_{L}\right)$ and gain factor $\left(K_{p}\right.$ and $\left.K_{n}\right)$ for both buffers are approximately equal. Therefore, adjusting the VDD will change the propagation delay, and a narrow pulse is formed by passing the two signals through an XOR gate. The adjustable voltage range is as shown in (10), which is dependent on the $V_{I H}$ specification of the selected components

$$
\begin{aligned}
t_{p} & =\frac{C_{L}}{2 V D D}\left(\frac{1}{k_{p}}+\frac{1}{k_{n}}\right) \\
\frac{V D D A}{\beta} & \geq V D D B \geq V_{I H, \mathrm{XOR}}, \quad \text { where } \beta \text { is } \frac{V_{I H, \text { buffers }}}{V D D B} .
\end{aligned}
$$

Apart from adjustable pulsewidth, the PRF and pulse amplitude can also be varied to meet the different operating requirements. The PRF is varied by changing the oscillator output, while adjustment of amplitude is performed by changing the VDDC. Fig. 15 shows the UWB pulse generated using the proposed pulse generator method. As seen, the generated pulse can be less than 1-ns wide.

\section{B. Analog Front-End and Microcontroller}

Typically ECG and EEG signals has amplitude of less than $500 \mu \mathrm{V}$ with frequency less than $100 \mathrm{~Hz}$. The front end of the transmitter uses an instrumental amplifier (INA321) and an active low-pass filter (LTC6081). The input signals are amplified by $60 \mathrm{~dB}$, INA321 produces a gain of $14 \mathrm{~dB}$, while LTC6081 provides a gain of $46 \mathrm{~dB}$ with a cutoff frequency at $100 \mathrm{~Hz}$. The 


\begin{tabular}{|c|c|c|c|c|c|c|c|c|c|c|}
\hline MSB & \multicolumn{9}{|c|}{ ADC Output } & LSB \\
\hline D9 & D8 & D7 & & D & & D4 & D3 & D2 & D1 & Do \\
\hline $\begin{array}{c}\text { Stop } \\
\text { bit }\end{array}$ & $\begin{array}{l}\text { Parity } \\
\text { bit }\end{array}$ & \multicolumn{2}{|l|}{ MSB } & \multicolumn{3}{|c|}{ Low Byte } & & & LSB & $\begin{array}{c}\text { Start } \\
\text { bit }\end{array}$ \\
\hline 1 & $\mathbf{P}$ & 1 & 0 & D5 & D4 & D3 & D2 & D1 & Do & 0 \\
\hline \multicolumn{11}{|c|}{ High Byte } \\
\hline 1 & $\mathbf{P}$ & 0 & \multicolumn{3}{|c|}{ Channel bits } & D9 & D8 & D7 & D6 & 0 \\
\hline
\end{tabular}

Fig. 16. Transmit data frame.

circuit is running from a $3.3-\mathrm{V}$ source with virtual ground set at $1.25 \mathrm{~V}$.

The microcontroller is operating with an external $20-\mathrm{MHz}$ crystal. Each analog front-end output is connected to one of the dedicated pins for the ADC module. The 10-bit ADC is programmed to sample at $4.8 \mathrm{kHz}$ with each channel sampling at $600 \mathrm{~Hz}$. The 10-bit data from each sample are separated into 2 B, as shown in Fig. 16. Low byte contains six least significant data bits (LSBs) and two header bits. High byte contains the four most significant data bits (MSBs), three channel bits, and one header bit. The MSB for the low byte is always " 1 " and "0" for the high byte. The three channel bits ranges from " 000 " to " 111 ," each representing one of the eight channels. High and low bytes are sent to the pulse generator through the universal asynchronous receiver/transmitter (UART) transmit pin, where a start bit, parity bit, and stop bit is added. The uutput data rate can range from $1.2 \mathrm{~kb} / \mathrm{s}$ to $1.25 \mathrm{Mb} / \mathrm{s}$. The maximum data rate is limited to $1.25 \mathrm{Mb} / \mathrm{s}$ because this is the highest UART baud rate that the microcontroller can support with a $20-\mathrm{MHz}$ crystal in our current prototype.

\section{Data Recovery for Low Data Rate UWB System}

Front ends for both the high data rate neural recording system and low data rate UWB-based wearable sensor system receivers are similar, but different data demodulation approaches are used for data recovery. A low data rate UWB transmitter sends multiple pulses per bit to increase the processing gain. The receiver is designed to sample at a rate much higher than the data rate. The information in the bit is determined, only after performing several samples; this increases the reliability of the system. The received signal after down conversion and low-pass filtering is converted to a digital signal using a 12-bit ADC embedded in the Stratix II development board. The demodulation is carried out with an FPGA chip running at $100 \mathrm{MHz}$. The receiver is capable of demodulating data of $50 \mathrm{Mb} / \mathrm{s}$.

The demodulation process is performed in two stages. In the first stage, the demodulator waits for the start signals to arrive, which is a low bit. Once the low bit is received, the demodulator samples the start bit until half of the period before sending a signal to start the demodulation clock. In the second stage, the demodulation clock goes high once the start signal is received. The first bit of data, which is the start bit, will be sent to the serial port. The demodulation clock runs at the same rate as the input data and samples the data at the middle of the bit. After the demodulation clock has generated 11 clock cycles, it goes low and wait for the start signal from stage one. The demodulated signal is transmitted through the serial port to the PC.

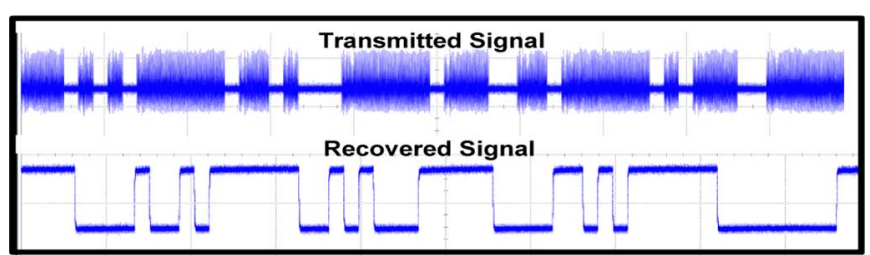

Fig. 17. Recovered data.

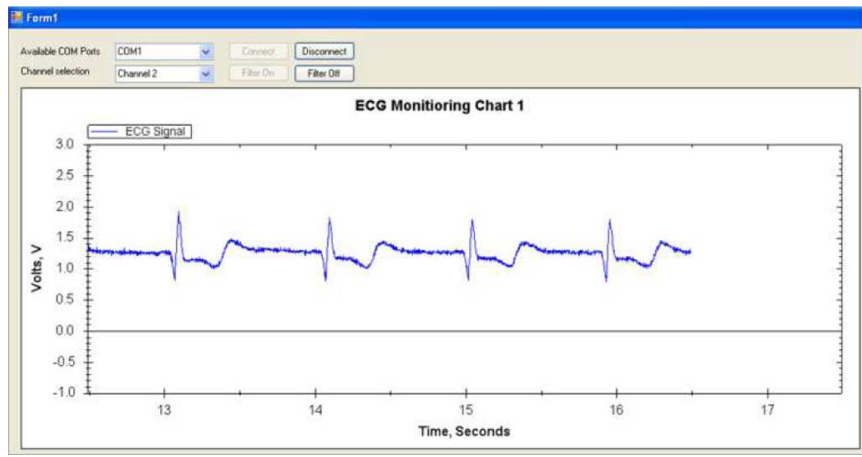

Fig. 18. Multichannel ECG signal.

The purpose of this demodulation structure is to ensure the data send to the PC is of the correct length to avoid any errors due to synchronization. This is necessary as multiple pulses per bit are sent, upon recovery there is a slight offset. The demodulated data is shown in Fig. 17. A program written using Visual Basic is used to decode the data; it performs filtering, as well as helps to displays the received signals on the screen. A parity bit check is performed on the received data to ensure all data received correctly. Once the received data is decoded, it is formatted back into a 10-bit word and separated based on the information embedded in the channel bits (see Fig. 16). Digital filtering is performed on the received signal to remove the $50 / 60-\mathrm{Hz}$ noise, which comes from the power supply. The ECG signal in Fig. 18 is successfully monitored in our laboratory environment with other wireless devices operating. The graphical user interface (GUI) program can display any eight channels by changing the button "channel selection" shown in the window.

\section{CONCLUSION}

A band-limited UWB pulse generation technique has been presented and analyzed. UWB transmitters based on the band-limited technique have been developed using both CMOS technology and from COTS components. The developed UWB transmitters are applied to low-power biomedical applications. The transmitters are suitable for both high data rate and low data rate applications. In an implantable environment, higher transmission power is required. For a low data rate system, there is a tradeoff between the processing gain and communication range. A multiple-channel EEG/ECG monitoring system using low data rate UWB technology has successfully been implemented and tested. The receiver in the prototype successfully received and recovered the UWB modulated ECG/EEG signals. The real time signals are displayed on a PC for body area network applications. 


\section{REFERENCES}

[1] G. A. Demichele and P. R. Troyk, "Integrated multichannel wireless biotelemetry system," in Proc. 25th IEEE EMBS Int. Conf., Cancun, Mexico, Sep. 17-21, 2003, pp. 3372-3375.

[2] I. Obeid, M. A. L. Nicolelis, and P. D. Wolf, "A multichannel telemetry system for single unit neural recordings," J. Neurosci. Meth., vol. 133, pp. 123-135, Feb. 2004.

[3] P. Mohseni and K. Najafi, "A battery-powered eight-channel wireless FM IC for biopotential recording applications," in Proc. IEEE Int. Solid-State Circuits Conf., San Francisco, CA, Feb. 6-10, 2005, pp. $560-561$.

[4] R. R. Harrison et al., "A low-power integrated circuit for a wireless 100-electrode neural recording system," IEEE J. Solid-State Circuits, vol. 42, no. 1, pp. 123-123, Jan. 2007.

[5] S. O'Driscoll, T. Meng, K. Shenoy, and C. Kemere, "Neurons to silicon: Implantable prosthesis processor," in IEEE Int. Solid-State Circuits Conf., San Francisco, CA, Feb. 2006, pp. 2248-2257.

[6] W. Liu, M. Sivaprakasam, G. Wang, and M. S. Chae, "A neural recording system for monitoring shark behavior," in Proc. IEEE Int. Circuits Syst. Symp., May 21-24, 2006, pp. 4123-4236.

[7] M. R. Yuce, W. Liu, M. S. Chae, and J. S. Kim, "A wideband telemetry unit for multi-channel neural recording systems," in IEEE Int. UltraWideband Conf., Sep. 24-26, 2007, pp. 612-617.

[8] M. Chae, W. Liu, Z. Yang, T. Chen, J. Kim, M. Sivaprakasam, and M. Yuce, "A 128-Channel $6 \mathrm{~mW}$ wireless neural recording IC with on-the-fly spike sorting and UWB transmitter," in Proc. IEEE SolidState Circuits Conf., Feb. 3-7, 2008, pp. 146-603.

[9] J. R. Ryckaert et al., "Ultra-wide band transmitter for low-power wireless body area networks: Design and evaluation," IEEE Trans. Circuits Syst. I, Reg. Papers, vol. 52, no. 12, pp. 2515-2515, Dec. 2005.

[10] D. D. Wentzloff and A. P. Chandrakasan, "Gaussian pulse generators for subbanded ultra-wideband transmitters," IEEE Trans. Microw. Theory Tech., vol. 54, no. 4, pp. 1647-1655, Apr. 2006.

[11] N. Deparis, C. Loyez, N. Rolland, and P.-A. Rolland, "Pulse generator for UWB communication and radar applications with PPM and time hopping possibilities," IEEE Int. Circuits Syst. Symp., pp. 661-665, 2006.

[12] P. Saha, N. Sasaki, and T. Kikkawa, "A single-chip Gaussian monocycle pulse transmitter using $0.18 \mu \mathrm{m}$ CMOS technology for intra/ interchip UWB communication," in IEEE VLSI Circuits Symp., Jun. 15-17, 2006, pp. 204-205.

[13] B. Jung, Y. H. Tseng, J. Harvey, and R. Harjani, "Pulse generator design for UWB IR communication systems," in IEEE Int. Circuits Syst. Symp., May 23-26, 2005, pp. 4381-4384.

[14] W. R. Bennett and J. R. Davey, Data Transmission. New York: McGraw-Hill, 1965.

[15] M. Chae, W. Liu, and M. Sivaprakasam, "Design optimization of integrated neural recording systems," IEEE J. Solid-State Circuits, vol. 43, no. 9, pp. 1931-1939, Sep. 2008.

[16] N. R. Mahapatra, A. Tareen, and S. V. Garimella, "Comparison and analysis of delay elements," in IEEE Midwest Circuits Syst. Symp., Aug. 2002, pp. 473-476.

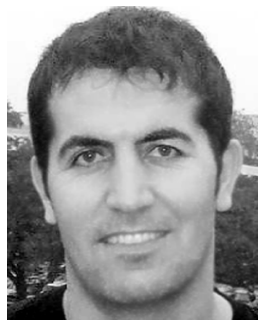

Mehmet Rasit Yuce (S'01-M'05) received the B.S. degree in electronics engineering from Ankara University, Ankara, Turkey, in 1997, the M.S. degree in electrical and computer engineering from the University of Florida, Gainesville, in 2001, and the Ph.D. degree in electrical and computer engineering from North Carolina State University (NCSU), Raleigh, in 2004

He is currently a Senior Lecturer with the School of Electrical Engineering and Computer Science, University of Newcastle, Callaghan, N.S.W., Australia. From August 2001 to October 2004, he was a Research Assistant with the Department of Electrical and Computer Engineering, NCSU. In 2005, he was a Post-Doctoral Researcher with the Electrical Engineering Department, University of California at Santa Cruz. He has authored or coauthored about 50 technical papers. His research interests include wireless implantable telemetry, wireless body area networks (WBANs), and analog/digital mixed signal very large scale integration (VLSI) for wireless, biomedical, and RF applications.

Dr. Yuce was the recipient of a National Aeronautics and Space Administration (NASA) Group Achievement Award in 2007 for the development of a silicon-on-insulator (SOI) transceiver.

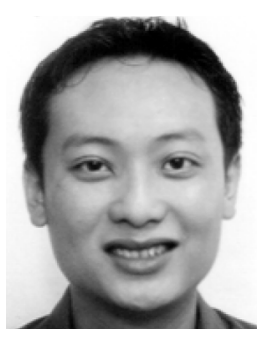

Ho Chee Keong received the B.S. degree in electrical engineering from the University of Newcastle, Callaghan, N.S.W., Australia, in 2006, and is currently working toward the Ph.D. degree at the University of Newcastle.

From 2006 to 2007, he was an Electronic Design Engineer with ET Designers, during which time he specialized in LCD and touch panel design. His research interests include UWB communication schemes, biomedical applications, and system designs for wireless communications.

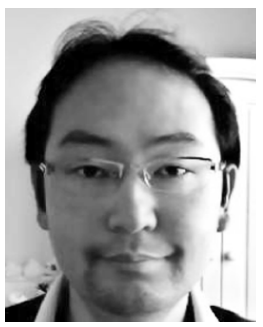

and retinal prostheses.
Moo Sung Chae was born on June 8, 1975 in Pusan, Korea. He received the B.S. and M.S. degrees in electrical engineering from Seoul National University, Seoul, Korea, in 1998 and 2000, respectively, and is currently working toward the Ph.D. degree at the University of California at Santa Cruz (UCSC).

In 2000, he joined Samsung Electronics, Seoul, Korea, where he was involved with circuit design of high-speed dynamic random access memories (DRAMs). His research interests are integrated neural recording systems, neural prosthetic devices, 